\title{
Kidney Matrix Stone in a Solitary Kidney
}

\author{
Heera Tharanendran ${ }^{1 *}$, Lakshmi Sundararajan ${ }^{1}$ and Ramesh Babu ${ }^{2}$
}

${ }^{1}$ Department of Pediatric Surgery, CHILDS Trust Medical Research Foundation, Kanchi Kamakoti CHILDS Trust Hospital, India

${ }^{2}$ Department of Pediatric Urology, Sri Ramachandra Institute of Higher Education \& Reasearch, India

ISSN: 2576-9200

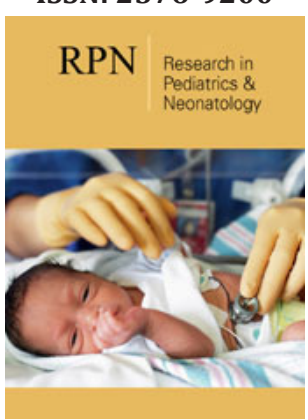

*Corresponding author: Heera Tharanendran, Department of Pediatric Surgery, CHILDS Trust Medical Research Foundation, Kanchi Kamakoti CHILDS Trust Hospital, Chennai, India

Submission: 㘹 February 02, 2021

Published: 柴March 26, 2021

Volume 5 - Issue 3

How to cite this article: Heera Tharanendran, Lakshmi Sundararajan, Ramesh Babu. Kidney Matrix Stone in a Solitary Kidney. Research in Pediatrics \& Neonatology. 5(3). RPN. 000615. 2021. DOI: $10.31031 / R P N .2021 .05 .000615$

Copyright $(\subset)$ Heera Tharanendran. This article is distributed under the terms of the Creative Commons Attribution 4.0 International License, which permits unrestricted use and redistribution provided that the original author and source are credited.

\section{Abstract}

A six-year-old boy with kidney matrix stones in solitary kidney is presented. Kidney matrix stones present in adults with atypical calcification on X-ray and CT, in the setting of recurrent urinary tract infections and instrumentation. These stones are not amenable to ESWL due to low calcium content. Complete stone clearance and prompt treatment of UTI are essential.

Keywords: Kidney; Matrix stones; Urolithiasis; Ureteroscopy; Pyelolithotomy; Children

\section{Introduction}

Kidney matrix stones are soft, pliable and amorphous calculi, reported only in adults [14]. We herein report a child with solitary kidney and recurrent renal calculi with atypical calcification on imaging identified as kidney matrix stones. This case is presented for its rarity and to create awareness about the entity.

\section{Case Report}

A six-year-old male child presented with acute renal failure and obstructive uropathy, elevated blood urea and serum creatinine, hyperkalemia, acidosis and urosepsis (elevated c-reactive protein and total count). The patient had undergone right nephrectomy and left pyelolithotomy two years earlier for bilateral staghorn calculi elsewhere. An X-ray abdomen showed multiple large calculi of varying size in left renal fossa with eggshell type calcification (Figure 1A).
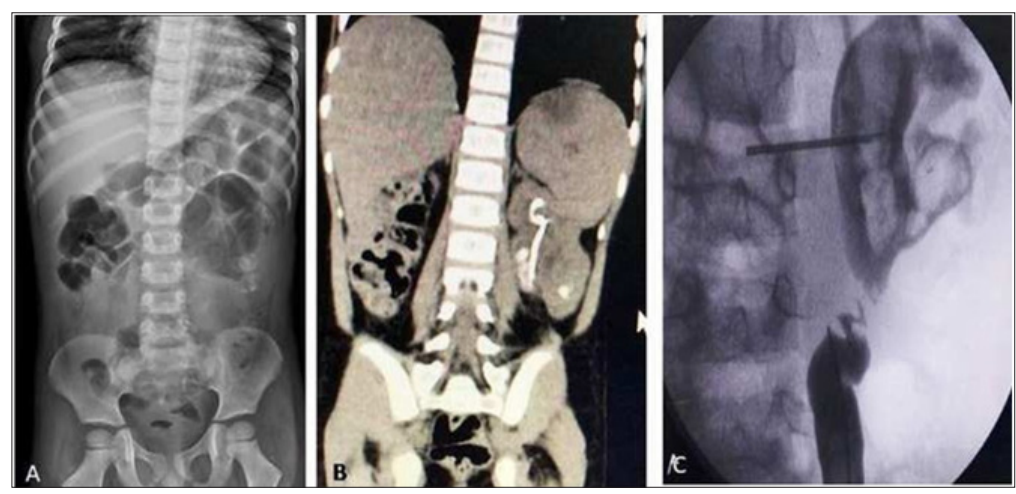

Figure 1:

A. Plain X-ray abdomen showing, radiolucent calculus with peripheral rim enhancement in the left renal pelvis and a radiopaque calculus in lower pole calyx

B. CT KUB showing heterogeneous incomplete stag horn calculus with denser calcification at the center and atypically low Hounsfield units (+120HU)

C. RGP showing a tight stricture at ureter pelvic junction.

Ultrasonogram (USG) of the abdomen revealed gross pyonephrosis of the solitary left kidney filled with multiple calculi. Patient was stabilised with a period of hydration, intra venous antibiotics and catheter drainage. Under general anesthesia a cystoscopic retrograde left Double-J (DJ) stent placement was performed; negotiation was difficult but succeeded with 
c-arm imaging. Computerised Tomography (CT) post-stabilisation showed heterogeneous incomplete calculus (Figure 1B) with denser calcification at the centre and atypically low Hounsfield units $(+120 \mathrm{HU})$. A percutaneous nephrolithotomy was planned but a Retro Grade Pyelogram (RGP) revealed a tight stricture at uretero pelvic junction (Figure 1C). Hence, open pyeloplasty, pyelolithotomy and antegrade stenting was performed. The retrieved stones were soft white and fleshy (Figure 2A) Histological assessment of the retrieved material showed lamellated appearance of pink amorphous acellular material (Figure 2B) with areas of calcification. Stone analysis revealed fine orthorhombic crystals of magnesium ammonium phosphate and protein material.
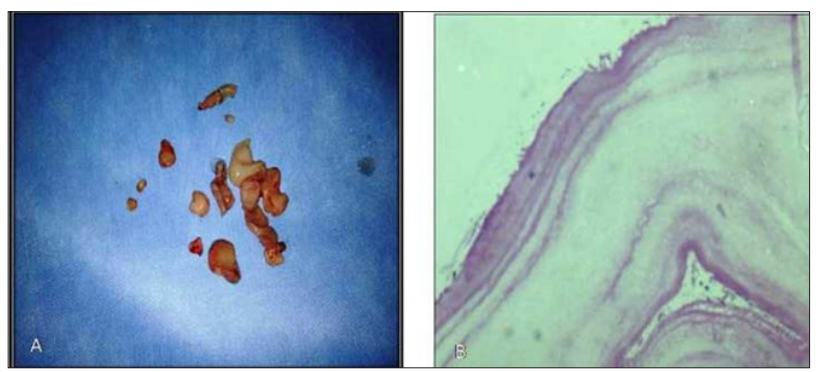

Figure 2:

A. Gross specimen showing Soft, white, fleshy mass

B. Histopathological examination showing Lamellated pink amorphous material.

At the time of planned DJ Stent removal after 6 weeks, an RGP showed a large recurrent filling defect in pelvis. Hence, rigid ureteroscopy was done, and the residual recurrent fleshy masses in the pelvis, which were extracted with grasper (Figure 3A). The matrix calculi appear fleshy like a white grape at ureteroscopy (Figure 3B). During the 6-month follow-up he passed some more whitish rounded fleshy mass in the urine exactly similar to what was seen at ureteroscopy. Repeat CT at 1 year follow up showed no residual calculi. A metabolic work up for stone disease was uncharacteristic.
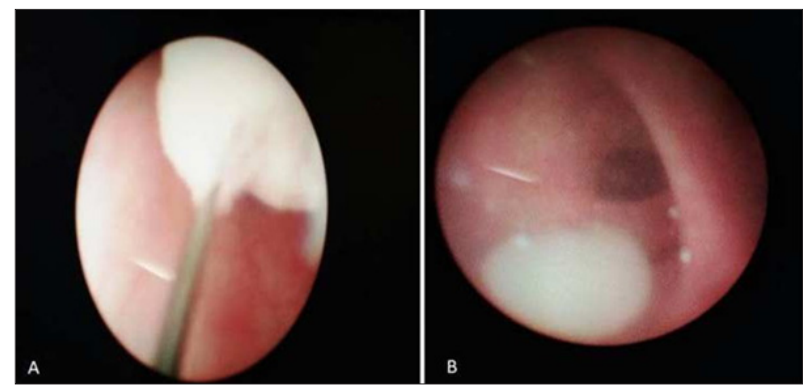

Figure 3: Rigid ureteroscopy showing

A. Recurrent fleshy mass in the pelvis being extracted with basket

B. White grape like structure represents matrix calculus.

\section{Discussion}

Renal matrix calculi are a rare subtype of kidney stones which were first reported 100 years ago by Gage and Beal1 and have also been called fibrinomas, colloid calculi, or albumin calculi. While more frequently encountered calcigerous stones tend to be brittle, matrix stones by contrast are soft, amorphous, and pliable. On average in this subtype of calculus, the noncrystalline, mucoprotein matrix accounts for $65 \%$ of its dry weight, compared to just $2.5 \%$ in more conventional stones [2]. Analysis by Boyce and Garvey 3 found the matrix to consist of mucopolysaccharide (one-third) and protein (two-thirds).

They have predominantly been reported in adult women. The main risk factors for this type of stones are previous surgery for stone disease and/or recurrent UTIs, especially due to Proteus mirabilis or Escherichia-coli [4]. Proteinuric patients with glomerulonephritis on dialysis are also at high risk of developing matrix calculi. The child in our report had also undergone multiple procedures previously for stone disease and had recurrent urinary tract infections.

The clinical presentation of patients with matrix stones is similar to those with calcigerous stones. Because of the soft consistency of matrix stones, they may conform to the shape of renal pelvis and ureter thereby causing gradual urinary obstruction and renal failure [5]. Singh $\mathrm{H}$ et al. [6] \& Matthews LA et al. [7] described cases of bilateral ureteric obstruction and acute renal failure secondary to bilateral matrix calculi. Patients can also rarely develop emphysematous Pyelonephritis [8].

Imaging appearances vary depending on the mineral composition and the pattern of distribution within the collecting system [9]. Plain X-ray in the present child showed a predominantly radiolucent stone with rim enhancement in the left pelvis, which on ultrasound showed a solid structure without the normal hyperechogenicity of stones and lacked post-acoustic shadowing. Unenhanced CT may identify these calculi which are described as having a mineral rim and soft tissue centre. In our case, CT showed heterogeneous calculi filling renal pelvis with low Hounsfield units $(+120 \mathrm{HU})$, which was very atypical for a calculus on CT imaging. Diagnostic uncertainty is common, especially where differential diagnoses for filling defects in adults include tumors [9]. On MRI, the matrix stones have hypointense signal in T1-weighted images, with no contrast enhancement following Gadolinium administration; T2-weighted images show a slight hyperintense signal. However, no specific radiological modality can definitively diagnose matrix stones and surgical intervention is frequently needed [9]. RGP can be used intra operatively as diagnostic tool [5]. Though we avoided RGP at the initial presentation in view of active infection and renal failure, RGP done after the acute episode showed tight stricture at PUJ and filling defects in the pelvicalyceal system. Endourological management is usually advocated; the choice lying between retrograde treatment and Percutaneous Nephrolithotomy (PCNL) depending on stone burden and location [8]. In our case we opted for an open procedure as there was associated PUJ stricture, which was dealt with open pyelothitomy and pyeloplasty. Retrograde ureterorenoscopy can be diagnostic in complex presentation as well as therapeutic for stone retrieval. In our case the recurrent stone was retrieved using rigid ureteroscope. 
For complete clearance of larger stones, PCNL is preferred as it has been shown to achieve excellent results with low recurrence rates. Extracorporeal Shock Wave Lithotripsy (ESWL) is usually ineffective due to the stone's gelatinous nature and a lack of breakable mineral content [10]. Fortunately, these stones have a very low recurrence rate once the stone is cleared completely [10]. Shah HN et al. [8] in his study stated that antibiotics for 4-6 weeks after PCNL, combined with RGP confirmation of stone clearance at the time of ureteric stent removal, accounts for the virtual absence of recurrent stones. To summarize kidney matrix calculi can pose diagnostic and therapeutic challenges and our case illustrates one such situation.

\section{References}

1. Gage H, Beal HW (1910) Fibrinous calculi in the kidney. Ann Surg 51(1): 111-112.

2. Allen TD, Spence HM (1996) Matrix stones. J Urol 95(3): 284-290.

3. Boyce WH, Garvey FK (1956) The amount and nature of the organic matrix in urinary calculi: A Review J Urol 76(3): 213-227.
4. Lahyani M, Rhannam Y, Slaoui A, Touzani A, Karmouni T, et al. (2016) Bilateral kidney matrix stones: A rare case. Pan Afr Med J 25: 102.

5. Bani-Hani AH, Segura JW, Leroy AJ (2005) Urinary matrix calculi: Our experience at a single institution. J Urol 173(1): 120-123.

6. Singh H, Pandey S, Dorairajan LN, Kumar S (2001) Acute renal failure due to bilateral matrix renal calculi--a diagnostic dilemma. Int Urol Nephrol 33(2): 311-313.

7. Matthews LA, Spirnak JP (1995) A matrix calculus causing bilateral ureteral obstruction and acute renal failure. J Urol 154(3): 1125-1126.

8. Shah HN, Kharodawala S, Sodha HS, Khandkar AA, Hegde SS, et al. (2009) The management of renal matrix calculi: A single-centre experience over 5 years. BJU Int 103(6): 810-814.

9. Cobley J, Phan YC, Mahmalji W (2018) Matrix stone occupying an entire renal collecting system: A case report and video of diagnostic ureterorenoscopy. Case Rep Urol 2018: 5892438.

10. Chan CHF, El-Hakim A, Andonian S (2010) Renal matrix stone managed by ureteroscopic holmium laser lithotripsy. Can J Urol 17(2): 5127-5130. 